\title{
Global Dynamics of an SIS Model on Metapopulation Networks with Demographics
}

\author{
Maoxing Liu $\mathbb{D},{ }^{1}$ Xinjie Fu $\mathbb{D},{ }^{1}$ Jie Zhang $\mathbb{D},{ }^{1}$ and Donghua Zhao $\mathbb{D}^{2}$ \\ ${ }^{1}$ Department of Mathematics, North University of China, Taiyuan 030051, Shanxi, China \\ ${ }^{2}$ School of Mathematical Science, Fudan University, Shanghai 200433, China \\ Correspondence should be addressed to Maoxing Liu; liumxsx@gmail.com
}

Received 16 August 2020; Revised 13 January 2021; Accepted 30 August 2021; Published 20 September 2021

Academic Editor: Sergio Gomez

Copyright (c) 2021 Maoxing Liu et al. This is an open access article distributed under the Creative Commons Attribution License, which permits unrestricted use, distribution, and reproduction in any medium, provided the original work is properly cited.

In this paper, we propose a susceptible-infected-susceptible (SIS) epidemic model with demographics on heterogeneous metapopulation networks. We analytically derive the basic reproduction number, which determines not only the existence of endemic equilibrium but also the global dynamics of the model. The model always has the disease-free equilibrium, which is globally asymptotically stable when the basic reproduction number is less than unity and otherwise unstable. We also provide sufficient conditions on the global stability of the unique endemic equilibrium. Numerical simulations are performed to illustrate the theoretical results and the effects of the connectivity and diffusion. Furthermore, we find that diffusion rates play an active role in controlling the spread of infectious diseases.

\section{Introduction}

In real life, the spread of infectious diseases is always affected by the flow of population. In order to study the impact of demographics and the flow of population, we will focus on an epidemic model with demographics on metapopulation networks. As Masuda [1] mentioned, underlying contact networks are considered to be static on the time scale of epidemics, whereas underlying humans' social networks of prevailing infectious diseases such as influenza are presumably dynamic even during one day. The dynamics of networks are induced by diffusion of individuals among residences, workplaces, places for social activities, and so on. As a simple framework within which the role of spatial processes in disease transmission can be examined, metapopulation models have been introduced to describe epidemics and ecological invasion in such a situation [1-3]. A node in such a model represents a metapopulation or a habitat and not an individual. A link represents a physical pathway connecting a pair of metapopulations. Individuals travel from one node to another. Simultaneously, interactions between individuals, such as infection, can occur in each metapopulation.
In recent years, the effect of diffusion on disease spread has caught the attention of many researchers [4-8]. Luca et al. [9] considered that metapopulation epidemic models can describe the spatial spread of an infectious disease through a spatially structured host population $[10,11]$. These models consist of patches or subpopulations of the system, connected through a coupling process generally characterizing hosts' mobility $[9,12]$. When the diffusion was first considered, the reaction-diffusion equation was regarded as a two-step process, and the reaction-diffusion was assumed to occur simultaneously to obtain the reaction-diffusion processes. Saldaña [13] derived continuous-time equations governing the limit dynamics of discrete-time reactiondiffusion processes defined on heterogeneous metapopulations, and the spread of infectious diseases under two different transmission mechanisms was considered. Juher et al. [14] proposed a system of continuous-time equations to analyze the spread of infectious diseases, which is based on the limited transmission and nonlimited transmission of diseases. Compared with reference [13], the authors studied the stability of endemic equilibrium in uncorrelated networks with nonlimited transmission. Therefore, a certain amount of diffusion is needed for the spread of diseases in 
metapopulation situation. After that, Masuda [1] investigated the effect of diffusion rate on the spread of epidemics in metapopulation networks. It can be seen from the above work that diffusion can change the epidemic threshold in many heterogeneous networks [1].

Based on previous work, we will investigate an epidemic model with demographics in metapopulation networks. Here the structure of patches (nodes) is made up of the connectivity (degree) distribution. Moreover, each patch contains two types of individuals: susceptible and infected individuals. Within each patch, transmission or recovery (reaction processes) occurred between individuals of different types. The diffusion of individuals takes place at once among patches (diffusion process) at constant rates. In fact, reaction and diffusion processes can be considered to take place simultaneously. With this idea, many epidemic models have been proposed to describe the dynamics of disease spread among patches. The model of this paper is a description of an SIS metapopulation model at the node level with birth and death. Based on the model in [1], we consider the model with node-based birth and death. Wang et al. [15] made use of a mean-field approximation, and this model has birth and death, but its birth and death do not change with the change of degree. In some models, in order to facilitate calculation, the elements of the connectivity matrix A are given by $a_{i i^{\prime}}=i p\left(i^{\prime}\right) /\langle i\rangle$. In this paper, we will analyze the model without using the mean-field approximation and transforming the elements of the adjacency matrix, and we study it in an arbitrary network.

The remaining of this paper is organized as follows. In Section 2, we firstly present the model and then study the existence of equilibria and their stability. There exist threshold dynamics that are determined by the basic reproduction number $R_{0}$. The theoretical results and the effects of connectivity and diffusion are demonstrated by numerical simulations in Section 3. The paper concludes with a summary and a brief discussion in last section.

\section{Main Results}

2.1. The Model. For a metapopulation network, each node stands for a metapopulation or patch. Let $N$ be the number of nodes; the $N \times N$ adjacency matrix, denoted by $A$, is defined by

$$
A_{j i}= \begin{cases}0, & \text { if } j=i \text { or } j \text { and } i \text { are not adjacent when } j \neq i, \\ 1, & \text { if } j \neq i \text { and } j \text { and } i \text { are adjacent. }\end{cases}
$$

Clearly, $A$ is symmetric. The model is an extension of the following one mentioned in Masuda [1]:

$$
\left\{\begin{array}{l}
\frac{\mathrm{d} \rho_{S, i}}{\mathrm{~d} t}=-\beta \rho_{S, i} \rho_{I, i}+\gamma \rho_{I, i}-D_{S} \rho_{S, i}+D_{S} \sum_{j=1}^{N} \frac{A_{j i}}{k_{j}} \rho_{S, j}, \\
\frac{\mathrm{d} \rho_{I, i}}{\mathrm{~d} t}=\beta \rho_{S, i} \rho_{I, i}-\gamma \rho_{I, i}-D_{I} \rho_{I, i}+D_{I} \sum_{j=1}^{N} \frac{A_{j i}}{k_{j}} \rho_{I, j},
\end{array}\right.
$$

where $\rho_{S, i}(t)$ and $\rho_{I, i}(t)$ represent the number of susceptible and infected individuals at node $i$ and at time $t$, respectively, $D_{S}$ and $D_{I}$ are the diffusion rates for the susceptible and infected individuals, respectively, $\beta$ is the transmission rate, $\gamma$ is the recovery rate, and $k_{i}$ is the degree of the node $i$. For (2), it is shown that diffusion increases the epidemic threshold of the SIS dynamics in arbitrary heterogeneous networks.

In this paper, we incorporate demographics into (2), and the model is as follows:

$$
\left\{\begin{array}{l}
\frac{\mathrm{d} \rho_{S, i}}{\mathrm{~d} t}=b_{i}-\beta \rho_{S, i} \rho_{I, i}+\gamma \rho_{I, i}-D_{S} \rho_{S, i}+D_{S} \sum_{j=1}^{N} \frac{A_{j i}}{k_{j}} \rho_{S, j}-\mu \rho_{S, i}, \\
\frac{\mathrm{d} \rho_{I, i}}{\mathrm{~d} t}=\beta \rho_{S, i} \rho_{I, i}-\gamma \rho_{I, i}-D_{I} \rho_{I, i}+D_{I} \sum_{j=1}^{N} \frac{A_{j i}}{k_{j}} \rho_{I, j}-\mu \rho_{I, i},
\end{array}\right.
$$

where $b_{i}$ is the birth rate at node $i$ and $\mu$ is the natural death rate of individuals (whose meaning is different from that in (2)).

It is easy to see that (3) is well posed, that is, for any given nonnegative initial condition, it has a unique and global solution through it. Moreover, the solution is also nonnegative. Let $\bar{b}=\sum_{i=1}^{N} b_{i}$ and $H=\sum_{i=1}^{N}\left(\rho_{S, i}+\rho_{I, i}\right)$. Adding up all equations of (3) yields

$$
\frac{\mathrm{d} H(t)}{\mathrm{d} t}=\bar{b}-\mu H(t)
$$

which implies that $\limsup _{t \rightarrow \infty} H(t) \leq(\bar{b} / \mu)$. Thus, the feasible region of (3) can be chosen as

$$
\Gamma=\left\{\left(\rho_{S, 1}, \ldots, \rho_{S, N}, \rho_{I, 1}, \ldots, \rho_{I, N}\right) \in R_{+}^{2 N} \mid \sum_{i=1}^{N}\left(\rho_{S, i}+\rho_{I, i}\right) \leq \frac{\bar{b}}{\mu}\right\} .
$$

It can be verified that $\Gamma$ is attractive and positively invariant with respect to (3) (see [16]). Let $\stackrel{\circ}{\Gamma}$ denote the interior of $\Gamma$ and $\partial \Gamma$ denote the boundary of $\Gamma$.

2.2. Existence of Disease-Free Equilibria. We start the analysis of (3) with the existence of disease-free equilibria. At a diseasefree equilibrium of (3), we have the following linear system [17]:

$$
b_{i}-D_{S} \rho_{S, i}+D_{S} \sum_{j=1}^{N} \frac{A_{j i}}{k_{j}} \rho_{S, j}-\mu \rho_{S, i}=0, \quad i=1,2, \ldots, N,
$$

which can be written in the matrix form

$$
\bar{L} S=\bar{B},
$$

where

$$
\bar{L}=\left(\begin{array}{cccc}
D_{S}+\mu & -D_{S} \frac{A_{21}}{k_{2}} & \cdots & -D_{S} \frac{A_{N 1}}{k_{N}} \\
-D_{S} \frac{A_{12}}{k_{1}} & D_{S}+\mu & \cdots & -D_{S} \frac{A_{N 2}}{k_{N}} \\
\vdots & \vdots & \ddots & \vdots \\
-D_{S} \frac{A_{1 N}}{k_{1}} & -D_{S} \frac{A_{2 N}}{k_{2}} & \cdots & D_{S}+\mu
\end{array}\right),
$$




$$
\begin{aligned}
S & =\left(\rho_{S, 1}, \rho_{S, 2}, \ldots, \rho_{S, N}\right)^{T}, \\
\bar{B} & =\left(b_{1}, b_{2}, \ldots, b_{N}\right)^{T} .
\end{aligned}
$$

As $\bar{L}$ is a column diagonal dominant matrix with positive diagonal elements and negative off-diagonal elements, $\bar{L}$ is nonsingular and any element of $\bar{L}^{-1}$ is positive [18]. Thus, (6) has a unique positive solution, which has the following result.

Proposition 1. System (3) always has a unique disease-free equilibrium, denoted by $E_{0}=\left(\rho_{S, 1}^{0}, \ldots, \rho_{S, N}^{0}, 0, \ldots, 0\right)$.

Since the adjacency matrix $A_{j i}$ is randomly given, the disease-free equilibrium $E_{0}$ given in Proposition 1 does not have an explicit expression. Thus, we calculate it in specific network: globally coupled network. In a globally coupled network,

$$
A=\left(\begin{array}{cccc}
0 & 1 & \cdots & 1 \\
1 & 0 & \cdots & 1 \\
\vdots & \vdots & \ddots & \vdots \\
1 & 1 & \cdots & 0
\end{array}\right)
$$

Then,

$$
\bar{L}=\left(\begin{array}{cccc}
D_{S}+\mu & -\frac{D_{S}}{N-1} & \cdots & -\frac{D_{S}}{N-1} \\
-\frac{D_{S}}{N-1} & D_{S}+\mu & \cdots & -\frac{D_{S}}{N-1} \\
\vdots & \vdots & \ddots & \vdots \\
-\frac{D_{S}}{N-1} & -\frac{D_{S}}{N-1} & \cdots & D_{S}+\mu
\end{array}\right) .
$$

By complex calculations, we get

$$
\bar{L}^{-1}=\left(\begin{array}{cccc}
\frac{(N-1) \mu+D_{S}}{N \mu D_{S}+\mu^{2}(N-1)} & \frac{D_{S}}{N \mu D_{S}+\mu^{2}(N-1)} & \cdots & \frac{D_{S}}{N \mu D_{S}+\mu^{2}(N-1)} \\
\frac{D_{S}}{N \mu D_{S}+\mu^{2}(N-1)} & \frac{(N-1) \mu+D_{S}}{N \mu D_{S}+\mu^{2}(N-1)} & \cdots & \frac{D_{S}}{N \mu D_{S}+\mu^{2}(N-1)} \\
\vdots & \vdots & \ddots & \vdots \\
\frac{D_{S}}{N \mu D_{S}+\mu^{2}(N-1)} & \frac{D_{S}}{N \mu D_{S}+\mu^{2}(N-1)} & \cdots & \frac{(N-1) \mu+D_{S}}{N \mu D_{S}+\mu^{2}(N-1)}
\end{array}\right),
$$

and hence

$\rho_{S, i}^{0}=\frac{\left[(N-1) \mu+D_{S}\right] b_{i}+D_{S}\left(-b_{i}+\sum_{j=1}^{N} b_{j}\right)}{N \mu D_{S}+\mu^{2}(N-1)}, \quad$ for $i=1,2, \ldots, N$.

In particular, if $b_{1}=b_{2}=\cdots=b_{N}=b$, then $\rho_{S, i}^{0}=(\mu / b)$ and the disease-free equilibrium is $E_{0}=(\underbrace{(\mu / b), \ldots,(\mu / b)}$, $0, \ldots, 0)$.
2.3. Stability of the Disease-Free Equilibrium. Before considering the stability of the disease-free equilibrium of (3), we introduce the basic reproduction number.

Linearizing (3) at the disease-free equilibrium $E_{0}$, we can get

$$
\frac{\mathrm{d} I(t)}{\mathrm{d} t}=M I(t)
$$

where $I(t)=\left(\rho_{I, 1}(t), \rho_{I, 2}, \ldots, \rho_{I, N}(t)\right)^{T}$ and

$$
M=\left(\begin{array}{cccc}
\beta \rho_{S, 1}^{0}-\gamma-\mu-D_{I} & D_{I} \frac{A_{21}}{k_{2}} & \cdots & D_{I} \frac{A_{N 1}}{k_{N}} \\
D_{I} \frac{A_{12}}{k_{1}} & \beta \rho_{S, 2}^{0}-\gamma-\mu-D_{I} & \cdots & D_{I} \frac{A_{N 2}}{k_{N}} \\
\vdots & \vdots & \ddots & \vdots \\
D_{I} \frac{A_{1 N}}{k_{1}} & D_{I} \frac{A_{2 N}}{k_{2}} & \cdots & \beta \rho_{S, N}^{0}-\gamma-\mu-D_{I}
\end{array}\right) .
$$


Then, $s(M)$ is a simple eigenvalue of $M$ with a positive eigenvector. Let $F_{i}(I)$ denote the rate of appearance of new infections in node $i, V_{i}^{+}(I)$ be the transfer rate of infected individuals into node $i$ by all other means, and $V_{i}^{-}(I)$ be the transfer rate of infected individuals out of node $i$. Then, we can choose

$$
\begin{gathered}
F=\left(\begin{array}{cccc}
\beta \rho_{S, 1}^{0} & 0 & \cdots & 0 \\
0 & \beta \rho_{S, 2}^{0} & \cdots & 0 \\
\vdots & \vdots & \ddots & \vdots \\
0 & 0 & \cdots & \beta \rho_{S, N}^{0}
\end{array}\right), \\
V=\left(\begin{array}{c}
V_{1}^{-}(I)-V_{1}^{+}(I) \\
V_{2}^{-}(I)-V_{2}^{+}(I) \\
\vdots \\
V_{N}^{-}(I)-V_{N}^{+}(I)
\end{array}\right)=\left(\begin{array}{cccc}
\mu+D_{I}+\gamma & -D_{I} \frac{A_{21}}{k_{2}} & \cdots & -D_{I} \frac{A_{N 1}}{k_{N}} \\
-D_{I} \frac{A_{12}}{k_{1}} & \mu+D_{I}+\gamma & \cdots & -D_{I} \frac{A_{N 2}}{k_{N}} \\
\vdots & \vdots & \ddots & \vdots \\
-D_{I} \frac{A_{1 N}}{k_{1}} & -D_{I} \frac{A_{2 N}}{k_{2}} & \cdots & \mu+D_{I}+\gamma
\end{array}\right) .
\end{gathered}
$$

We call $F V^{-1}$ the next generation matrix for (3), and the basic reproduction number $R_{0}$ is defined as

$$
R_{0}=\rho\left(F V^{-1}\right)
$$

where $\rho\left(F V^{-1}\right)$ denotes the spectral radius of the matrix $F V^{-1}$ (see [19] for details). Note that $R_{0}$ is simply the product of the infection rate and the mean duration of the infection [15]. In a globally coupled network, the basic reproduction number is $R_{0}=(b \beta / \mu(\gamma+\mu))$. Refer to [19] for Theorem 1 .

Theorem 1. The following statements on (3) are true.

(i) $R_{0}>1$ if and only if $s(M)>0$ and $R_{0}<1$ if and only if $s(M)<0$.

(ii) If $R_{0}<1$, then the disease-free equilibrium $E_{0}$ is locally asymptotically stable while it is unstable if $R_{0}>1$.

Now, we establish the global stability of the disease-free equilibrium.

Theorem 2. Assume $R_{0}<1$. Suppose that $A$ is irreducible. Then, the disease-free equilibrium $E_{0}$ of (3) is globally asymptotically stable in $R_{+}^{2 N}$.

Proof. As $\Gamma$ is an attracting and positively invariant set, we only need to show the global stability of $E_{0}$ in $\Gamma$.

Let $F$ and $V$ be given by (15) and (16), respectively. Since all off-diagonal entries of $V$ are nonpositive and the sum of the entries in each column of $V$ is positive, $V$ is a nonsingular $M$-matrix. Also, $V^{-1}$ is irreducible. By the PerronFrobenius theorem ([18], p. 27), the nonnegative irreducible matrix $V^{-1} F$ has a positive left eigenvector $\left(w_{1}, w_{2}, \ldots, w_{N}\right)$ corresponding to the eigenvalue $\rho\left(V^{-1} F\right)$. Since $F$ is a diagonal matrix, $\rho\left(V^{-1} F\right)=\rho\left(F V^{-1}\right)$. Consequently, we have

$$
\left(w_{1}, w_{2}, \ldots, w_{N}\right) V^{-1} F=R_{0}\left(w_{1}, w_{2}, \ldots, w_{N}\right),
$$

or

$$
\frac{1}{R_{0}}\left(w_{1}, w_{2}, \ldots, w_{N}\right)=\left(w_{1}, w_{2}, \ldots, w_{N}\right) F^{-1} V .
$$

For $i=1, \ldots, N$, denote $c_{i}=\left(w_{i} / \beta \rho_{S, i}^{0}\right)>0$ and $I=\left(\rho_{I, 1}, \rho_{I, 2}, \ldots, \rho_{I, N}\right)^{T}$. Consider the Lyapunov function

$$
L=\sum_{i=1}^{N} c_{i} \rho_{I, i}
$$

and the derivative of $L$ along the solutions of (3) is

$$
\begin{aligned}
\left.\frac{\mathrm{d} L}{\mathrm{~d} t}\right|_{(2.2)} & =\sum_{i=1}^{N} c_{i}\left(\beta \rho_{S, i} \rho_{I, i}-\gamma \rho_{I, i}-D_{I} \rho_{I, i}+D_{I} \sum_{j=1}^{N} \frac{A_{j i}}{k_{j}} \rho_{I, j}-\mu \rho_{I, i}\right) \\
& \leq \sum_{i=1}^{N} c_{i}\left(\beta \rho_{S, i}^{0}-\gamma-\mu-D_{I}\right) \rho_{I, i}+D_{I} \sum_{j \neq i}^{N} \frac{A_{j i}}{k_{j}} \rho_{I, j} \\
& =\left(\frac{w_{1}}{\beta \rho_{S, 1}^{0}}, \frac{w_{2}}{\beta \rho_{S, 2}^{0}}, \ldots, \frac{w_{N}}{\beta \rho_{S, N}^{0}}\right)(F-V) I \\
& =\left(w_{1}, w_{2}, \ldots, w_{N}\right)\left(1-F^{-1} V\right) I \\
& =\left(w_{1}, w_{2}, \ldots, w_{N}\right)\left(1-\frac{1}{R_{0}}\right) I \leq 0 .
\end{aligned}
$$


Since $c_{i}>0$ for all $i,\left.(\mathrm{~d} L / \mathrm{d} t)\right|_{(2,2)}=0$ implies that either $\rho_{S, i}=\rho_{S, i}^{0}$ or $\rho_{I, i}=0$ for any $1 \leq i \leq N$. When $\rho_{S, i}=\rho_{S, i}^{0}$, it follows from the first equation of (3) that

$$
0=\frac{\mathrm{d} \rho_{S, i}^{0}}{\mathrm{~d} t}=b_{i}-\beta \rho_{S, i}^{0} \rho_{I, i}+\gamma \rho_{I, i}-D_{S} \rho_{S, i}^{0}+D_{S} \sum_{j=1}^{N} \frac{A_{j i}}{k_{j}} \rho_{S, j}^{0}-\mu \rho_{S, i}^{0} .
$$

Comparing this with (6) gives $\rho_{I, i}=0$. Thus, we have shown that $\left.(\mathrm{d} L / \mathrm{d} t)\right|_{(2.2)}=0$ which implies that $\rho_{I, i}=0$ for all $i$. By LaSalle's invariance principle, we see that $E_{0}$ is globally asymptotically stable in $\Gamma$ and hence in $R_{+}^{2 N}$.

The following result tells us that when $R_{0}>1$, the disease is persistent.

Proposition 2 (see [17]). Suppose that $A$ is irreducible. If $R_{0}>1$, then system (3) is uniformly persistent and there exists an endemic equilibrium $E^{*}$ in $\stackrel{\circ}{\Gamma}$.

The proof of Proposition 2 is similar to the proof of Proposition 3.3 in [20]. Using uniform persistence of (3) and uniform boundedness of solutions in $\stackrel{\circ}{\Gamma}$ we can obtain the existence of an equilibrium of (3) in $\stackrel{\Gamma}{\Gamma}$ (see Theorem 2.8.6 in [21]). In Proposition 2, the assumption that the adjacency matrix $A=\left(A_{i j}\right)$ is irreducible is necessary. If $A=0$, then system (3) has an asymptotically stable boundary equilibrium when $R_{0}>1$, and thus the system is not persistent [22].
2.4. The Global Stability of the Endemic Equilibrium. In this section, we provide sufficient conditions on the global stability of the endemic equilibrium. The method of proof is the graph-theoretical approach developed in [23-25].

Theorem 3. Assume that $R_{0}>1$. Let $E^{*}=\left(\rho_{S, 1}^{*}, \ldots, \rho_{S, N}^{*}, \rho_{I, 1}^{*}, \ldots, \rho_{I, N}^{*}\right)$ be an endemic equilibrium of (3). If $A$ is irreducible and there exists $\lambda>0$ such that $D_{S} \sum_{j=1}^{N}\left(A_{j i} / k_{j}\right) \rho_{S, j}^{*}=\lambda D_{I} \sum_{j=1}^{N}\left(A_{j i} / k_{j}\right) \rho_{I, j}^{*} \quad$ for all $1 \leq i, j \leq N$, then the endemic equilibrium $E^{*}$ is globally asymptotically stable in $\stackrel{\circ}{\Gamma}$, and hence (3) only has a unique endemic equilibrium.

Proof. For each $1 \leq i \leq N$, set

$$
V_{i}\left(\rho_{S, i}, \rho_{I, i}\right)=\rho_{S, i}-\rho_{S, i}^{*}-\rho_{S, i}^{*} \ln \frac{\rho_{S, i}}{\rho_{S, i}^{*}}+\rho_{I, i}-\rho_{I, i}^{*}-\rho_{I, i}^{*} \ln \frac{\rho_{I, i}}{\rho_{I, i}^{*}} .
$$

Noting

$$
\begin{array}{r}
D_{S} \rho_{S, i}^{*}=b_{i}-\beta \rho_{S, i}^{*} \rho_{I, i}^{*}+\gamma \rho_{I, i}^{*}+D_{S} \sum_{j=1}^{N} \frac{A_{j i}}{k_{j}} \rho_{S, j}^{*}-\mu \rho_{S, i}^{*}, \\
\left(D_{I}+\mu\right) \rho_{I, i}^{*}=\beta \rho_{S, i}^{*} \rho_{I, i}^{*}+D_{I} \sum_{j=1}^{N} \frac{A_{j i}}{k_{j}} \rho_{I, j}^{*}-\gamma \rho_{I, i}^{*},
\end{array}
$$

we calculate the derivative of $V_{i}$ along solutions of (3) as

$$
\begin{aligned}
&\left.\frac{\mathrm{d} V_{i}}{\mathrm{~d} t}\right|_{(2.2)}= b_{i}-D_{S} \rho_{S, i}+D_{S} \sum_{j=1}^{N} \frac{A_{j i}}{k_{j}} \rho_{S, j}-\mu \rho_{S, i}-b_{i} \frac{\rho_{S, i}^{*}}{\rho_{S, i}}+\beta \rho_{I, i} \rho_{S, i}^{*} \\
&-\gamma \rho_{I, i} \frac{\rho_{S, i}^{*}}{\rho_{S, i}}+D_{S} \rho_{S, i}^{*}-D_{S} \sum_{j=1}^{N} \frac{A_{j i}}{k_{j}} \rho_{S, j} \frac{\rho_{S, i}^{*}}{\rho_{S, i}}+\mu \rho_{S, i}^{*}-D_{I} \rho_{I, i} \\
&+D_{I} \sum_{j=1}^{N} \frac{A_{j i}}{k_{j}} \rho_{I, j}-\mu \rho_{I, i}-\beta \rho_{S, i} \rho_{I, i}^{*}+\gamma \rho_{I, i}^{*}+D_{I} \rho_{I, i}^{*}+\mu \rho_{I, i}^{*}-D_{I} \sum_{j=1}^{N} \frac{A_{j i}}{k_{j}} \rho_{I, j} \rho_{I, i}^{*} \\
& \rho_{I, i} \\
&= b_{i}\left[1-\frac{\rho_{S, i}}{\rho_{S, i}^{*}}+\ln \frac{\rho_{S, i}}{\rho_{S, i}^{*}}+1-\frac{\rho_{S, i}^{*}}{\rho_{S, i}}+\ln \frac{\rho_{S, i}^{*}}{\rho_{S, i}}\right] \\
&+D_{S} \sum_{j=1}^{N} \frac{A_{j i}}{k_{j}} \rho_{S, j}^{*}\left[1-\frac{\rho_{S, i}^{*} \rho_{S, j}}{\rho_{S, i} \rho_{S, j}^{*}}+\ln \frac{\rho_{S, i}^{*} \rho_{S, j}}{\rho_{S, i} \rho_{S, j}^{*}}\right] \\
&+D_{S} \sum_{j=1}^{N} \frac{A_{j i}}{k_{j}} \rho_{S, j}^{*}\left[\frac{\rho_{S, j}}{\rho_{S, j}^{*}}+\ln \frac{\rho_{S, j}^{*}}{\rho_{S, j}}-\frac{\rho_{S, i}}{\rho_{S, i}^{*}}-\ln \frac{\rho_{S, i}^{*}}{\rho_{S, i}}\right] \\
&+D_{I} \sum_{j=1}^{N} \frac{A_{j i}}{k_{j}} \rho_{I, j}^{*}\left[1-\frac{\rho_{I, i}^{*} \rho_{I, j}}{\rho_{I, i} \rho_{I, j}^{*}}+\ln \frac{\rho_{I, i}^{*} \rho_{I, j}}{\rho_{I, i} \rho_{I, j}^{*}}\right]
\end{aligned}
$$




$$
\begin{aligned}
& +D_{I} \sum_{j=1}^{N} \frac{A_{j i}}{k_{j}} \rho_{I, j}^{*}\left[\frac{\rho_{I, j}}{\rho_{I, j}^{*}}+\ln \frac{\rho_{I, j}^{*}}{\rho_{I, j}}-\frac{\rho_{I, i}}{\rho_{I, i}^{*}}-\ln \frac{\rho_{I, i}^{*}}{\rho_{I, i}}\right] \\
& +\gamma \rho_{I, i}^{*}\left[1-\frac{\rho_{S, i}^{*} \rho_{I, i}}{\rho_{S, i} \rho_{I, i}^{*}}+\ln \frac{\rho_{S, i}^{*} \rho_{I, i}}{\rho_{S, i} \rho_{I, i}^{*}}\right]+\gamma \rho_{I, i}^{*}\left[\ln \frac{\rho_{I, i}^{*}}{\rho_{I, i}}-\ln \frac{\rho_{S, i}^{*}}{\rho_{S, i}}\right] \\
& +\gamma \rho_{I, i}\left[1-\frac{\rho_{I, i}^{*} \rho_{S, i}}{\rho_{I, i} \rho_{S, i}^{*}}+\ln \frac{\rho_{I, i}^{*} \rho_{S, i}}{\rho_{I, i} \rho_{S, i}^{*}}\right]+\gamma \rho_{I, i}\left[\ln \frac{\rho_{I, i}}{\rho_{I, i}^{*}}-\ln \frac{\rho_{S, i}}{\rho_{S, i}^{*}}\right] \\
& \leq D_{S} \sum_{j=1}^{N} \frac{A_{j i}}{k_{j}} \rho_{S, j}^{*}\left[\frac{\rho_{S, j}}{\rho_{S, j}^{*}}+\ln \frac{\rho_{S, j}^{*}}{\rho_{S, j}}-\frac{\rho_{S, i}}{\rho_{S, i}^{*}}-\ln \frac{\rho_{S, i}^{*}}{\rho_{S, i}}\right] \\
& +D_{I} \sum_{j=1}^{N} \frac{A_{j i}}{k_{j}} \rho_{I, j}^{*}\left[\frac{\rho_{I, j}}{\rho_{I, j}^{*}}+\ln \frac{\rho_{I, j}^{*}}{\rho_{I, j}}-\frac{\rho_{I, i}}{\rho_{I, i}^{*}}-\ln \frac{\rho_{I, i}^{*}}{\rho_{I, i}}\right] \\
& +\gamma \rho_{I, i}^{*}\left[\ln \frac{\rho_{I, i}^{*}}{\rho_{I, i}}-\ln \frac{\rho_{S, i}^{*}}{\rho_{S, i}}\right]+\gamma \rho_{I, i}\left[\ln \frac{\rho_{I, i}}{\rho_{I, i}^{*}}-\ln \frac{\rho_{S, i}}{\rho_{S, i}^{*}}\right] \\
& =D_{S} \sum_{j=1}^{N} \frac{A_{j i}}{k_{j}} \rho_{S, j}^{*}\left[\frac{\rho_{S, j}}{\rho_{S, j}^{*}}+\ln \frac{\rho_{S, j}^{*}}{\rho_{S, j}}-\frac{\rho_{S, i}}{\rho_{S, i}^{*}}-\ln \frac{\rho_{S, i}^{*}}{\rho_{S, i}}\right] \\
& +D_{I} \sum_{j=1}^{N} \frac{A_{j i}}{k_{j}} \rho_{I, j}^{*}\left[\frac{\rho_{I, j}}{\rho_{I, j}^{*}}+\ln \frac{\rho_{I, j}^{*}}{\rho_{I, j}}-\frac{\rho_{I, i}}{\rho_{I, i}^{*}}-\ln \frac{\rho_{I, i}^{*}}{\rho_{I, i}}\right] \\
& =D_{I} \sum_{j=1}^{N} \frac{A_{j i}}{k_{j}} \rho_{I, j}^{*}\left(\lambda \frac{\rho_{S, j}}{\rho_{S, j}^{*}}+\lambda \ln \frac{\rho_{S, j}^{*}}{\rho_{S, j}}+\frac{\rho_{I, j}}{\rho_{I, j}^{*}}+\ln \frac{\rho_{I, j}^{*}}{\rho_{I, j}}\right) \\
& -D_{I} \sum_{j=1}^{N} \frac{A_{j i}}{k_{j}} \rho_{I, j}^{*}\left(\lambda \frac{\rho_{S, i}}{\rho_{S, i}^{*}}+\lambda \ln \frac{\rho_{S, i}^{*}}{\rho_{S, i}}+\frac{\rho_{I, i}}{\rho_{I, i}^{*}}+\ln \frac{\rho_{I, i}^{*}}{\rho_{I, i}}\right) \\
& =D_{I} \sum_{j=1}^{N} \frac{A_{j i}}{k_{j}} \rho_{I, j}^{*}\left[G_{j}\left(\rho_{S, j}, \rho_{I, j}\right)-G_{i}\left(\rho_{S, i}, \rho_{I, i}\right)\right] \text {, }
\end{aligned}
$$

where

$$
G_{i}\left(\rho_{S, i}, \rho_{I, i}\right)=\lambda \frac{\rho_{S, i}}{\rho_{S, i}^{*}}+\lambda \ln \frac{\rho_{S, i}^{*}}{\rho_{S, i}}+\frac{\rho_{I, i}}{\rho_{I, i}^{*}}+\ln \frac{\rho_{I, i}^{*}}{\rho_{I, i}} .
$$

Here we have used the fact that $1-x+\ln x \leq 0$ for $x>0$, and the equality holds if and only if $x=1$.

Consider a weight matrix $W=\left(w_{i j}\right)$ with entries $w_{i j}=$ $D_{I}\left(A_{j i} / k_{j}\right) \rho_{I, j}^{*}$ and denote the corresponding weighted digraph as $(G, W)$. Let $l_{i}=\sum_{T \in \mathbb{T}_{i}} w(T) \geq 0$ be the same as that given in (A.1) in the Appendix associated with $(\mathscr{G}, W)$. Then, by (A.2), the following identity holds:

$$
\sum_{i=1}^{N} l_{i} \sum_{j=1}^{N} D_{I} \frac{A_{j i}}{k_{j}} \rho_{I, j}^{*}\left[G_{j}\left(\rho_{S, j}, \rho_{I, j}\right)-G_{i}\left(\rho_{S, i}, \rho_{I, i}\right)\right]=0 .
$$

Set

$$
V\left(\rho_{S, 1}, \rho_{I, 1}, \ldots, \rho_{S, N}, \rho_{I, N}\right)=\sum_{i=1}^{N} l_{i} V_{i}\left(\rho_{S, i}, \rho_{I, i}\right) .
$$

Then, we obtain

$$
\begin{aligned}
\left.\frac{\mathrm{d} V}{\mathrm{~d} t}\right|_{(2.2)} & =\left.\sum_{i=1}^{N} l_{i} \frac{\mathrm{d} V_{i}}{\mathrm{~d} t}\right|_{(2.2)} \\
& \leq \sum_{i=1}^{N} l_{i} \sum_{j=1}^{N} D_{I} \frac{A_{j i}}{k_{j}} \rho_{I, j}^{*}\left[G_{j}\left(\rho_{S, j}, \rho_{I, j}\right)-G_{i}\left(\rho_{S, i}, \rho_{I, i}\right)\right]=0,
\end{aligned}
$$

for all $\left(\rho_{S, 1}, \rho_{I, 1}, \ldots, \rho_{S, N}, \rho_{I, N}\right) \in \stackrel{\circ}{\Gamma}$. Since $A$ is irreducible, we know that $l_{i}>0$ for all $i$ (see the Appendix), and thus $\left.(\mathrm{d} V / \mathrm{d} t)\right|_{(2.2)}=0$ implies that $\rho_{S, i}=\rho_{S, i}^{*}$ for all $i$. From the first equation of (3), we obtain

$$
0=b_{i}-\beta \rho_{S, i}^{*} \rho_{I, i}+\gamma \rho_{I, i}-D_{S} \rho_{S, i}^{*}+D_{S} \sum_{j=1}^{N} \frac{A_{j i}}{k_{j}} \rho_{S, j}^{*}-\mu \rho_{S, i}^{*},
$$

for $i=1, \ldots, N$, which implies that $\rho_{I, i}=\rho_{I, i}^{*}$. This means that the largest invariant set in $\left\{\left.(\mathrm{d} V / \mathrm{d} t)\right|_{(2.2)}=0\right\}$ is the singleton $\left\{E^{*}\right\}$. By LaSalle's invariance principle, $E^{*}$ is globally asymptotically stable in $\stackrel{\circ}{\Gamma}$. 


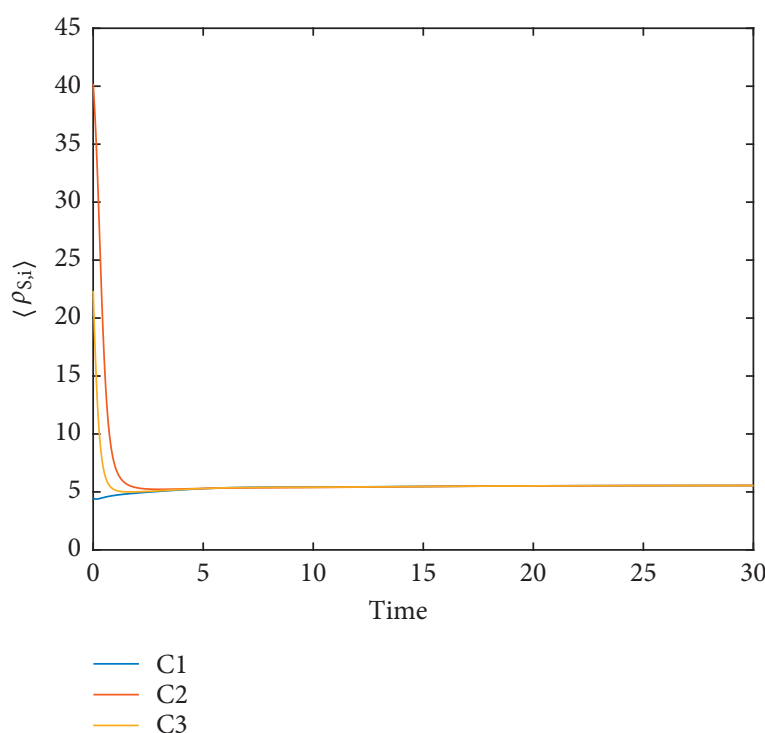

(a)

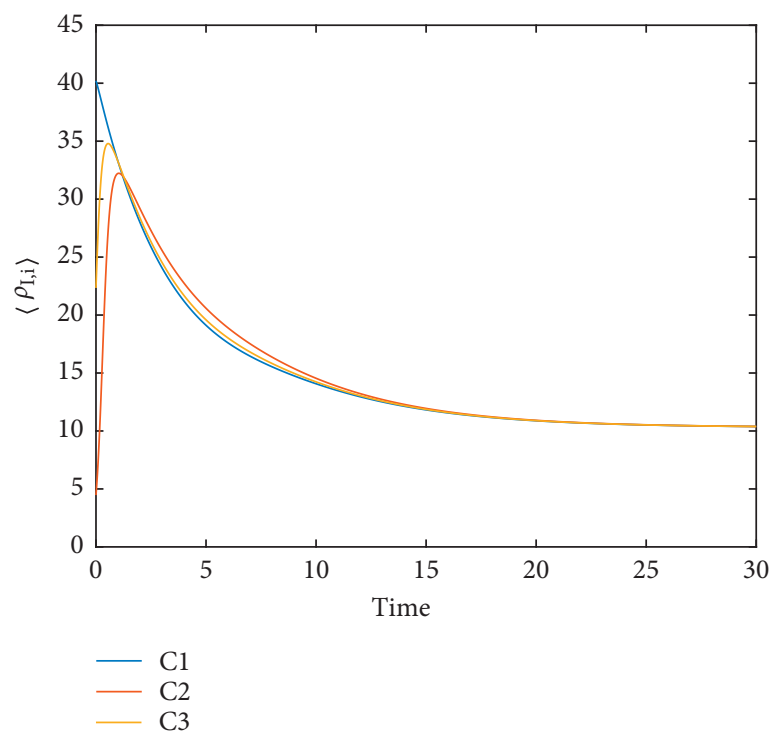

(b)

FIGURE 1: The average number of susceptible and infected individuals with different initial values.

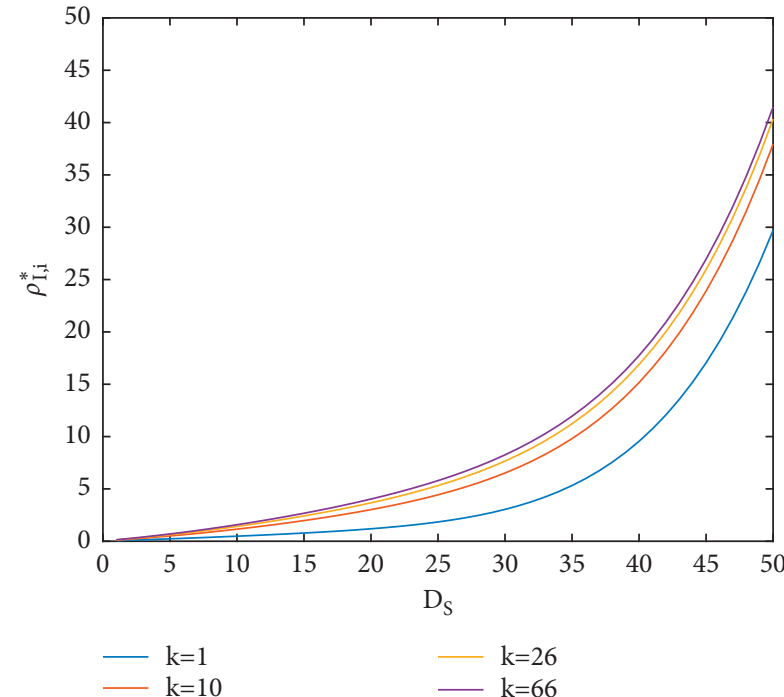

(a)

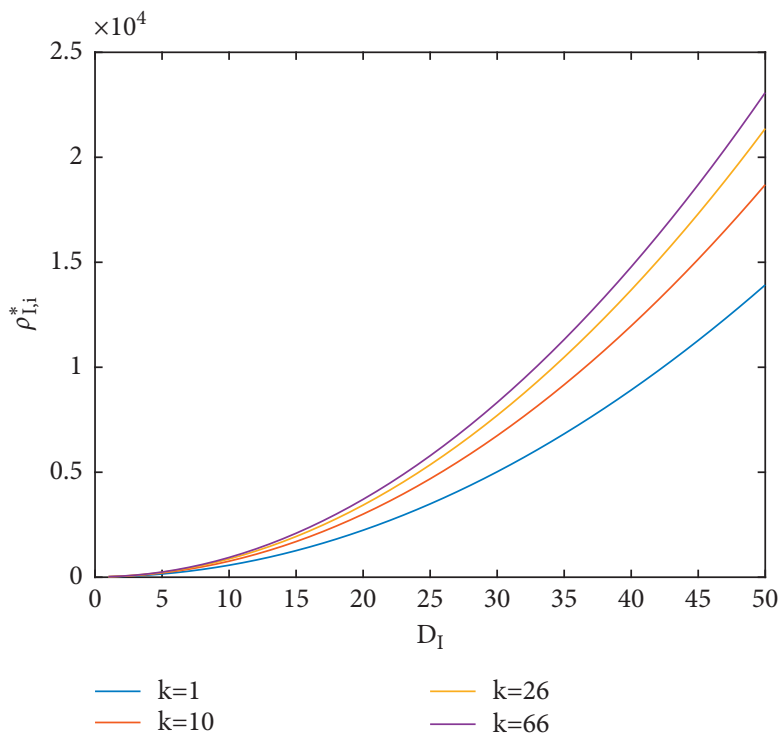

(b)

FIGURE 2: The impact of diffusion on the number of infected individuals at the endemic equilibria, and each line corresponds to the nodes with degrees $k$ of $1,10,26$, and 66, respectively (from bottom to top). (a) Relationship between $D_{S}$ and $\rho_{I, i}^{*}$. (b) Relationship between $D_{I}$ and $\rho_{I, i}^{*}$.

\section{Numerical Simulations}

The results in the previous section presented the stability of the disease-free and endemic equilibria. Through numerical simulations of infection dynamics, it is shown that the connectivity between patches has a large effect on disease dynamics. In this section, an Erdös-Rényi network with 100 nodes is established to study the influence of diffusion, and the connection probability is 0.4 . All parameters are positive.
It should be noted that we simulated the numerical solution of the model equation, not Monte Carlo (MC) simulations of the metapopulation dynamics [14].

Figure 1 shows the average number of susceptible and infected individuals with different initial values. The parameters are used as $N=100, b=1, \mu=0.00312, \beta=0.125$, $D_{S}=0.2, D_{I}=0.2$, and $\gamma=0.6$. We select three different initial conditions for simulation, and the three initial values were $\mathrm{C} 1$ : the ratio of susceptible number to infected number 


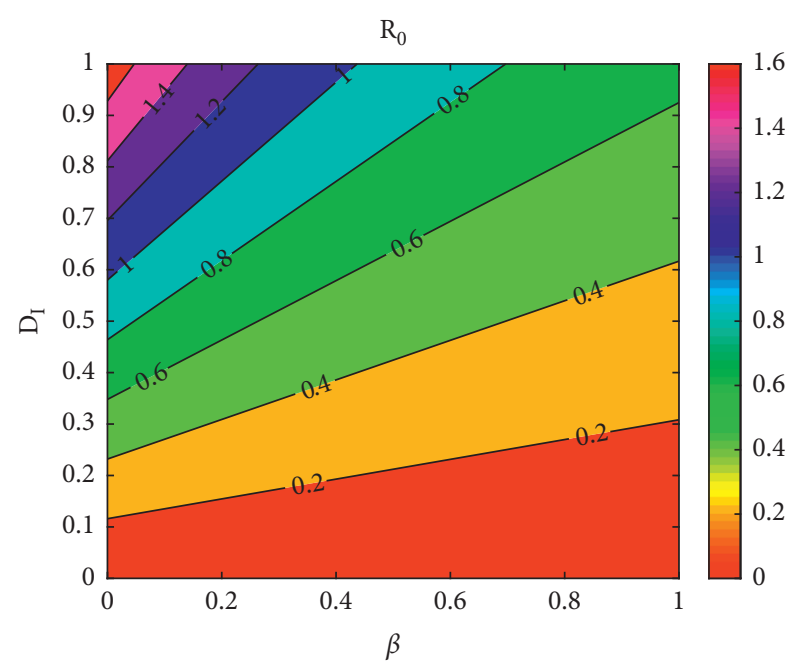

(a)

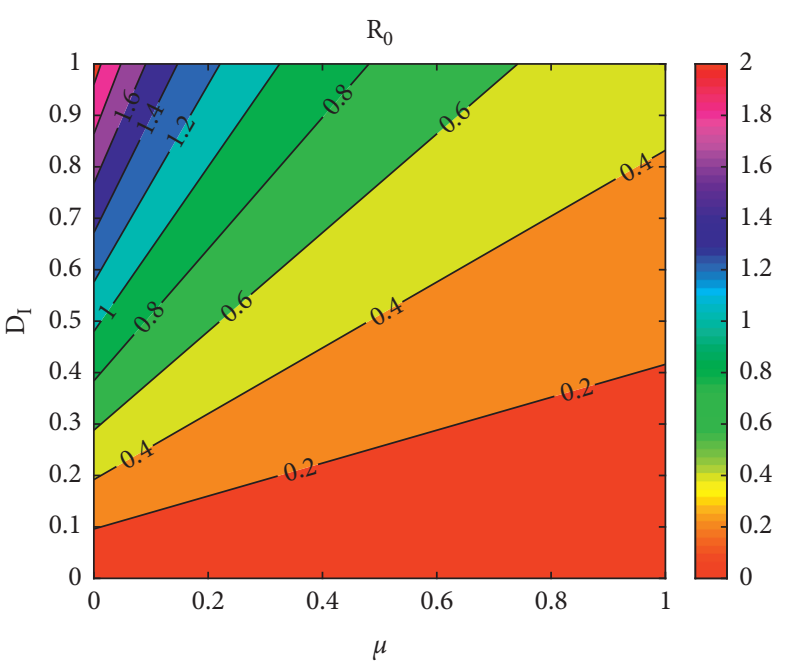

(b)

Figure 3: The impact of diffusion and other parameters on $R_{0}$. (a) Relationship between $\beta, D_{I}$, and $R_{0}$. (b) Relationship between $\mu, D_{I}$, and $R_{0}$.

is 1:9; C2: the ratio of susceptible number to infected number is $1: 1$; and C3: the ratio of susceptible number to infected number is $9: 1$. Susceptible and infected individuals obey Poisson distribution in nodes. It can be seen in Figure 1 that under different initial conditions, both susceptible individuals and infected individuals converge to a unique globally asymptotically stable endemic equilibrium.

In Figure 2, we consider the impact of diffusion on the number of infected individuals at the endemic equilibrium. For this purpose, we take $N=100, b=6, \mu=0.00512$, $\beta=0.325$, and $\gamma=0.6$. In Figure 2(a), we fix $D_{I}=1$. We observe that $\rho_{I, i}^{*}$ increases as $D_{S}$ increases and for the same $D_{S}$, when $i$ is larger, $\rho_{I, i}^{*}$ is also larger. In Figure 2(b), we take $D_{S}=2$. It is observed that the same phenomena happen with respect to $D_{I}$ and $i$ with fixed $D_{I}$.

The relationship between $\beta, D_{I}$, and $R_{0}$ is simulated in Figure 3(a), and the relationship between $\mu, D_{I}$, and $R_{0}$ is simulated in Figure 3(b). It can be seen from Figure 3 that the influence of $D_{I}$ on $R_{0}$ is greater than that of $\beta$ and $\mu$, so the influence of the diffusion rates for the infected individuals on disease outbreak cannot be ignored.

\section{Conclusion}

In this paper, we formulated and analyzed an SIS model with demographics and diffusion on metapopulation networks. In this model, we solved the stability of the disease-free equilibrium and deduced the basic reproduction number. We concluded that when $R_{0}<1$, the disease-free equilibrium is globally asymptotically stable; otherwise, it is unstable. Next, we proved the existence and stability of endemic equilibria. The endemic equilibrium is globally asymptotically stable when $R_{0}>1$. Finally, we gave some numerical simulations to illustrate the main results and demonstrate the effects of connectivity and diffusion on the number of infected individuals at the endemic equilibrium. As the connectivity or diffusion is increasing, the number of infected individuals is also increasing. This means that when an epidemic comes, the more people there are and the greater the degree is, the more likely the disease will break out. Therefore, we should pay attention to the prevention and control of diseases and make plans in advance.

The research of this paper can be extended to the following aspects: in this paper, the Erdős-Rényi network is used to simulate, and the models based on other networks can be analyzed; secondly, the infectious disease model with heterogeneous diffusion rate can also be analyzed. It is necessary to study the mathematical model under a specific network in the future, so as to make the model more realistic.

\section{Appendix}

\section{A. Combinatorial Identity}

Let $(\mathscr{G}, W)$ be a weighted digraph with $N(>2)$ vertices, where $W=\left(w_{i j}\right)$ is the weight matrix. A weight $w_{i j}>0$ if the directed arc $(j, i)$ from vertex $j$ to vertex $i$ exists; otherwise, $w_{i j}=0$. Let $\mathbb{T}_{i}$ be the set of all spanning trees of $(\mathscr{G}, W)$ rooted at vertex $i$. For $T \in \mathbb{T}_{i}$, the weight of $T$, denoted by $w(T)$, is the product of weights on all arcs of $T$. Let

$$
l_{i}=\sum_{T \in \mathbb{T}_{i}} w(T), \quad i=1,2, \ldots, N .
$$

Then, $l_{i} \geq 0$ and

$$
\sum_{i, j=1}^{N} l_{i} w_{i j} G_{i}\left(x_{i}\right)=\sum_{i, j=1}^{N} l_{i} w_{i j} G_{j}\left(x_{j}\right),
$$

for any family of functions $\left\{G_{i}\left(x_{i}\right)\right\}_{i=1}^{N}$. If $W=\left(w_{i j}\right)$ is irreducible, then $l_{i}>0$ for $i=1,2, \ldots, N$. We refer readers to [22] for the proof of (A.1). 


\section{Data Availability}

The data used to support the findings of this study are available from the corresponding author upon request.

\section{Conflicts of Interest}

The authors declare that there are no conflicts of interest regarding the publication of this paper.

\section{Acknowledgments}

This study was supported by the National Natural Science Foundation of China (nos. 12071445 and 12001501), Fund for Shanxi (1331KIRT), Shanxi Natural Science Foundation (nos. 201801D221033 and 201901D211216), Graduate Innovation Project in Shanxi Province (2020BY104), and the Outstanding Youth Fund of North University of China.

\section{References}

[1] N. Masuda, "Effects of diffusion rates on epidemic spreads in metapopulation networks," New Journal of Physics, vol. 12, 2010093009.

[2] L. Longini and I. L. Jr, "A mathematical model for the global spread of influenza," Mathematical Biosciences, vol. 75, no. 1, pp. 3-22, 1985.

[3] I. Hanski and O. Ovaskainen, "The metapopulation capacity of a fragmented landscape," Nature, vol. 404, no. 6779, pp. 755-758, 2000.

[4] S. Bonaccorsi, S. Ottaviano, D. Mugnolo, and F. D. Pellegrini, "Epidemic outbreaks in networks with equitable or almostequitable partitions," SIAM Journal on Applied Mathematics, vol. 75, no. 6, pp. 2421-2443, 2015.

[5] V. Colizza and A. Vespignani, "Epidemic modeling in metapopulation systems with heterogeneous coupling pattern: theory and simulations," Journal of Theoretical Biology, vol. 251 , no. 3 , pp. $450-467,2008$

[6] C. Poletto, S. Meloni, V. Colizza, Y. Moreno, and A. Vespignani, "Host mobility drives pathogen competition in spatially structured populations," PLoS Computational Biology, vol. 9, no. 8, Article ID 1003169, 2013.

[7] K. M. A. Ksbir and J. Tanimoto, "Evolutionary vaccination game approach in metapopulation migration model with information spreading on different graphs," Chaos, Solitons \& Fractals, vol. 120, pp. 41-55, 2019.

[8] W. Wang, X. Gao, Y. Cai, H. Shi, and S. Fu, "Turing patterns in a diffusive epidemic model with saturated infection force," Journal of the Franklin Institute, vol. 355, no. 15, pp. 72267245, 2018.

[9] G. Luca, K. V. Kerckhove, P. Coletti et al., "The impact of regular school closure on seasonal influenza epidemics: a data-driven spatial transmission model for Belgium," BMC Infectious Diseases, vol. 18, no. 1, 29 pages, 2018.

[10] I. Hanski and O. E. Gaggiotti, "Ecology, genetics and evolution of metapopulations," Journal of the Torrey Botanical Society, vol. 131, no. 4, 2004.

[11] M. J. Keeling and P. Rohani, "Estimating spatial coupling in epidemiological systems: a mechanistic approach," Ecology Letters, vol. 5, no. 1, pp. 20-29, 2002.

[12] A. Ewing, E. C. Lee, C. Viboud, and S. Bansal, "Contact, travel, and transmission: the impact of winter holidays on influenza dynamics in the United States," Journal of Infectious Diseases, vol. 215, no. 5, pp. 732-739, 2017.

[13] J. Saldaña, "Continuous-time formulation of reaction-diffusion processes on heterogeneous metapopulations," Physical review. E, Statistical, nonlinear, and soft matter physics, vol. 78, Article ID 012902, 2008.

[14] D. Juher, J. Ripoll, and J. Saldaña, “Analysis and Monte Carlo simulations of a model for the spread of infectious diseases in heterogeneous metapopulations," Physical review. E, Statistical, nonlinear, and soft matter physics, vol. 80, Article ID 041920, 2009.

[15] J. Wang, M. Liu, and Y. Li, "Analysis of epidemic models with demographics in metapopulation networks," Physica A: Statistical Mechanics and Its Applications, vol. 392, no. 7, pp. 1621-1630, 2013.

[16] L. Perko, Differential Equations and Dynamical Systems, Springer-Verlag, Berlin, Germany, 2000.

[17] M. Y. Li and Z. Shuai, "Global stability of an epidemic model in a patchy environment," Canadian Applied Mathematics Quarterly, vol. 17, no. 1, pp. 175-187, 2009.

[18] A. Berman and R. J. Plemmons, Nonnegative Matrices in the Mathematical Sciences, Academic Press, Cambridge, MA, USA, 1979.

[19] P. van den Driessche and J. Watmough, "Reproduction numbers and sub-threshold endemic equilibria for compartmental models of disease transmission," Mathematical Biosciences, vol. 180, no. 1-2, pp. 29-48, 2002.

[20] M. Y. Li, J. R. Graef, L. Wang, and J. Karsai, "Global dynamics of a SEIR model with varying total population size," Mathematical Biosciences, vol. 160, no. 2, pp. 191-213, 1999.

[21] N. P. Bhatia and G. P. Szego, Dynamical Systems: Stability Theory and Applications, Springer-Verlag, Berlin, Germany, 1967.

[22] F. Brauer, P. van den Driessche, and L. Wang, "Oscillations in a patchy environment disease model," Mathematical Biosciences, vol. 215, no. 1, pp. 1-10, 2008.

[23] H. Guo, M. Y. Li, and Z. Shuai, "Global stability of the endemic equilibrium of multigroup SIR epidemic models," Canadian Applied Mathematics Quarterly, vol. 14, pp. 259284, 2006

[24] H. Guo, M. Y. Li, and Z. Shuai, "A graph-theoretic approach to the method of global Lyapunov functions," Proceedings of the American Mathematical Society, vol. 136, no. 8, pp. 2793-2802, 2008.

[25] M. Y. Li and Z. Shuai, "Global-stability problem for coupled systems of differential equations on networks," Journal of Differential Equations, vol. 248, no. 1, pp. 1-20, 2010. 